\title{
Real-time aircraft noise likeness detector
}

\author{
C. Asensio*, M. Ruiz, M. Recuero \\ Universidad Politécnica de Madizd, Grupo de Investigación en Instrumentación y Acística Aplicada (I2A2), INSIA - Ctra. Vatencia km 7, 28031 Madird, Spain
}

\section{A R T I C L E I N F O}

Keywords:

Aircraft noise

Monitoring

Pattern recognition

One-class classification

Detection

\begin{abstract}
A B S T R A C T
One of the most difficult tasks involved in the process of noise monitoring near airports is related to the automatic detection and classification of aircraft noise events.

These tasks can be solved by applying pattern recognition techniques to the audio signal captured by a microphone. But now the problem is caused by the background noise, which is present in real environments.

This paper proposes a real-time method for continuously tracking the similarity of the input sound and the aircraft's sounds. Using these facilities, the monitoring unit will be able to mark aircraft events, or to make measurements only when aircraft sound is louder than background noise.

A one-class approach has been applied to this detection-by-classification method.

Using the default setup, 93\% of the aircraft's events which held an SNR of 6-8 dB were detected, for 30 different locations with diverse soundscapes.
\end{abstract}

\section{Introduction}

Noise pollution has become one of the most important reasons for concern related to environment in advanced countries. In order to manage and reduce noise pollution and its adverse effects, some legislation initiatives have been carried out (the most important is Directive 2002/49/EC [1]).

Referring to transport infrastructures, noise monitoring systems are very important for planning stages and noise management.

Noise monitoring units, especially those integrated into airport monitoring systems, must be able to measure sound level time history, identifying sound events and classifying the events produced by aircraft.

The classification task, in relation to the sound source of the noise event, is the weak point of the monitoring units.

A simple monitoring unit detects noise events using thresholds applied to sound level time history. When noise level reaches a value over the threshold for longer than an established duration, a noise event is detected.

The classification of these events, in most of the monitoring systems of international airports is carried out using radar tracks of flights $[2,3]$. If the noise event has been detected when an aircraft is near the monitor, this event will be classified as produced by an aircraft. No other acoustic characteristics of the sound are considered.

Some other attempts have been made to classify environmental noise events. They used pattern recognition or speech recognition techniques in order to classify environmental sound events [4-10]. Most of them applied a detection and classification approach, using neural networks [11], hidden Markov models [11,12], source separation [13], They have shown quite good results, but the main problem they have shown is related to detection in noisy environments.

Many other references can be found related to speech or speaker recognition, auditory scene classification, musical instruments. The compilation of the main works in non-speech sound events detection and classification can be found in Refs. $[14,15]$

Explicitly related to aircraft sounds recognition [16,17], apply neural networks for the recognition of airplane type during taking off.

Although pattern recognition approaches are still used [18], nowadays, most of the research strategies in the detection of environmental noise sources have been oriented to the use of microphone arrays [19].

\section{Objectives}

The main objective of this project is to design a system that can detect aircraft sounds in real time, so that its integration with a monitoring unit can improve aircraft detection rates during unattended measurements.

The input signal will be acquired by a single microphone, which could be the one in the monitoring unit.

This system will be especially useful in standalone monitors which are not integrated into an airport monitoring system (when no radar data are available). 


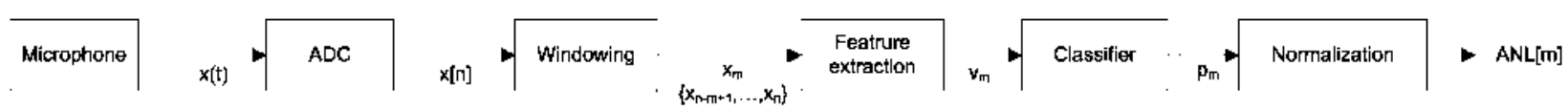

Fig. 1. ANL derection scheme.

\section{Methodology}

\subsection{Audio recordings}

The first stage in this project consisted in gathering sound recordings and acoustic measurements at different locations in order to make a description of the noise sources for different soundscapes, with a large number of different aircraft and non-aircraft sounds, in noisy and quiet environments, in the presence and the absence of aircraft.

Thirty different outdoors locations were selected in several citjes in Spain, most of them near Madrid-Barajas airport.

A noise monitor, equipped with an outdoor microphone, was used to $\log$ equivalent sound pressure level every second ( $\left.L_{\text {Aeq.15 }}\right)$. The AC output of the monitor was also recorded in approximately one hour length audio files $(44,100 \mathrm{~Hz}, 16$ bits, mono). The audio files were synchronized with the measurement logs in order to track the sound events.

The main acoustic events (aircraft and non-aircraft) in the recordings were manually labeled and recorded in new files. The measurement files were also marked, and measured. The results in this processing and the labeled audio files were included in a database to allow a correct management during the rest of the project.

More than 1000 aircraft events (approximately $14 \mathrm{~h}$ ), and 1700 non-aircraft audio files ( $36 \mathrm{~h}$ ) were labeled.

\subsection{Aircraft noise likeness}

The noise generated by aircraft is one of those sounds that the human ear can recognize almost anywhere, no matter what the duration of the sound or prior knowledge about the probability of flyovers in that location.

Although sight, perceived sound source location or other information can be useful, the audio signal acquired by a single microphone has most of the knowledge needed for recognizing aircraft sounds. Because of this, it is possible to detect aircraft sounds indoors or in movies, for instance.

The method proposed in this paper for the detection of aircraft sounds is based on the similarity between the input sound and the sound (noise) generated by aircraft.

By applying a kind of simple fuzzy sets theory [20], an ANL index has been defined (aircraft noise likeness), to track the similarity between the input sound and generic aircraft sounds. ANL is the grade of membership of the instantaneous sound input to the aircraft noise class. The ANL value of 1 denotes full membership, pure aircraft noise. As the input is corrupted by background noise, the ANL value decreases, and the weaker the membership in the aircraft sound fuzzy set.

\subsection{Real-time ANL detection}

ANL must be calculated from the audio signal acquired by a microphone, using the scheme in Fig. 1.
This is a classical scheme in pattern recognition. After a digitization and windowing process, feature vectors must be extracted.

Previous works [4] have shown that the best results in the recognition of environmental sounds can be obtained by using Mel frequency coefficients (MFCC). So we decided to exploit MFCC (13 coefficients), but using an extended bandwidth (starting at $0 \mathrm{~Hz}$ ), which have shown better performance due to the low frequency componелts іл aircraft noise [21].

Fig. 2 shows the scheme used for feature extraction [22].

To perform the classification task, we used PRTools [23] for Matlab. A statistical classifier continuously receives the input patterns and updates its output, according to the Bayes decision rule [24].

$P\left(C_{1} \| x\right)>p\left(C_{2} \| x\right)$ then Class1, Class2.

where $P\left(C_{0} \| x\right)$ is the posteriori probability of the pattern $x$ to belong to class $C_{n}$.

Instead of using a classical classifier crisp output (1 or 2 , for classes $C_{1}$ or $C_{2}$ ), a sigmoid has been applied for normalization of its soft outputs, so ANL is calculated as the probability of the input pattern belonging to the class aircraft sound.

\subsection{One-class approach}

The first attempt to face the classification task was made using the classical two class pattern recognition approach. The first class was supposed to be aircraft noise, and the second one non-aircrafts sounds.

Good results were obtained for the first tests, as few soundscapes were used for the training of the system. But when trying to generalize results, and testing the system for larger acoustic environments, we found that error rates increased.

Fig. 3 shows the probability distribution for the thirteen features in classes aircraft (target), and non-aircraft (outliers).

Although we had tried to get many objects from the non-aircraft class, it is not possible to make a good generalization, as it will always be possible to find new non-aircraft sounds that were not considered during the training of the classifier.

In order to minimize the effect of a bad sampling of the non-aircraft class, we tried a one-class classification approach [25-27], which has shown itself to be more effective. This approach was applied by [28] to the classification of sounds.

This new approach has proved to be very effective when only one of the classes can be described with precision, because only a few samples are available, or because of the huge amount of different objects involved. For instance, this approach has shown good results for the detection of faces in an image [29].

The training an implementation of the classifier has been carried out using data description toolbox for Matlab [30].

A mixture of Gaussians classifier was selected because of its performance (speed and error rates). As we had many outliers available, the model was adjusted according to the following equation
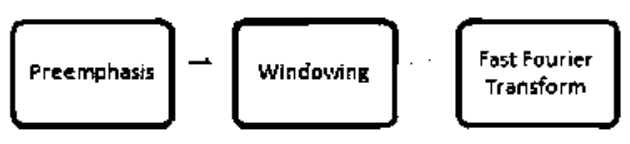
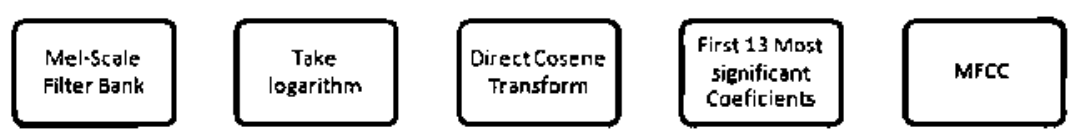

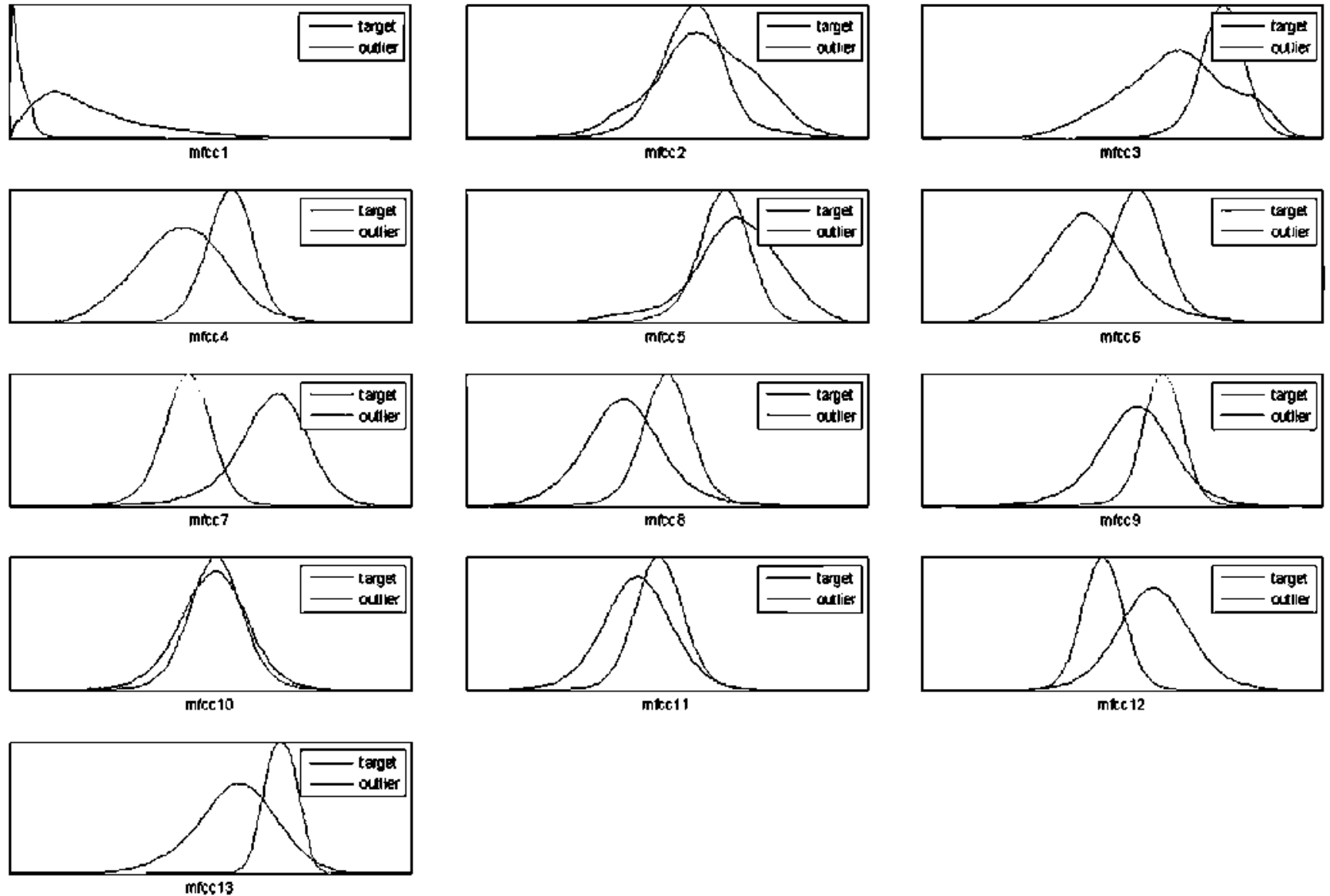

Fig. 3. Probability density function of features in classes aircraft (target) and non-aircraft (outlier).

$$
\begin{aligned}
f(x)= & \sum_{i=1}^{K t} P_{i} \exp \left(-\left(x-\mu_{i}\right)^{T} \Sigma_{i}^{-1}\left(x-\mu_{i}\right)\right) \\
& -P_{*} \exp \left(-(x-\mu)^{T} \Sigma_{*}^{-1}(x-\mu)\right) \\
& -\sum_{i=1}^{K 0} P_{j} \exp \left(-\left(x-\mu_{j}\right)^{T} \Sigma_{j}^{-1}\left(x-\mu_{j}\right)\right)
\end{aligned}
$$

where the first line stands for the target class distribution, the third stands for the outliers distribution, and the second line stands for a 'background outlier' (see [30] for details). Then the classifier would be $h(x)$ as follows:

$$
h(x)=\left\{\begin{array}{l}
\text { target } \text { if } f(x) \geqslant \theta \\
\text { outlier if } f(x)<\theta
\end{array}\right.
$$

The classifier can be adjusted for a better fit to the target class dataset, but, during the training, a threshold must be defined to set the allowed error in the target class.

Testing the classifier on the available outlier objects, and varying the threshold, it is possible to find the performance of the classifier on the outlier class. This is the receiver operating characteristics curve (ROC-curve). By analyzing the ROC-curve, it is possible to decide the classifier's working point.

Fig. 4 shows the ROC-curve for three models: 5,10 and 20 Gaussians per class. For every rate of targets accepted we were able to configure as the classifier working point, the 20 Gaussian model accepts a lower rate of outliers.

During the training, a $10 \%$ error in targets (for training dataset) was chosen. Although it is not really important for this application. the model had $11 \%$ of rejected targets and $7 \%$ of accepted outliers when applied to the test dataset.

This adjusted and trained model is used to get the ANL index. The classifier's soft output is normalized to express the probability of the input vector being a target. This is the crisp ANL, defined as follows

$\operatorname{ANL}(x)=p($ Target $\| x)$

\subsection{Data exploitation}

When the first training set was created, all locations and conditions were used indistinctly. Polluted aircraft sound files were not included, but no other distinctions were made.

The results were quite poor because the noise sources were not distributed uniformly, so some sources had many samples while others had very few.

Additionally, the starting and finishing fragments of aircraft audio files were included in training, so highly polluted samples were considered as targets. In a few cases, this caused the detection of aircraft that did not really exist in the recording, and, in many cases, this caused the detection of nearly imperceptible aircraft sounds, which could not be viewed in the measurements profile, and should not be measured.

A data exploitation and selective sampling process [31-33] had to be made in order to optimize results:

- Only fragments of 'pure' aircraft noise were selected to create the target class dataset (starting and finishing fragments were rejected). 


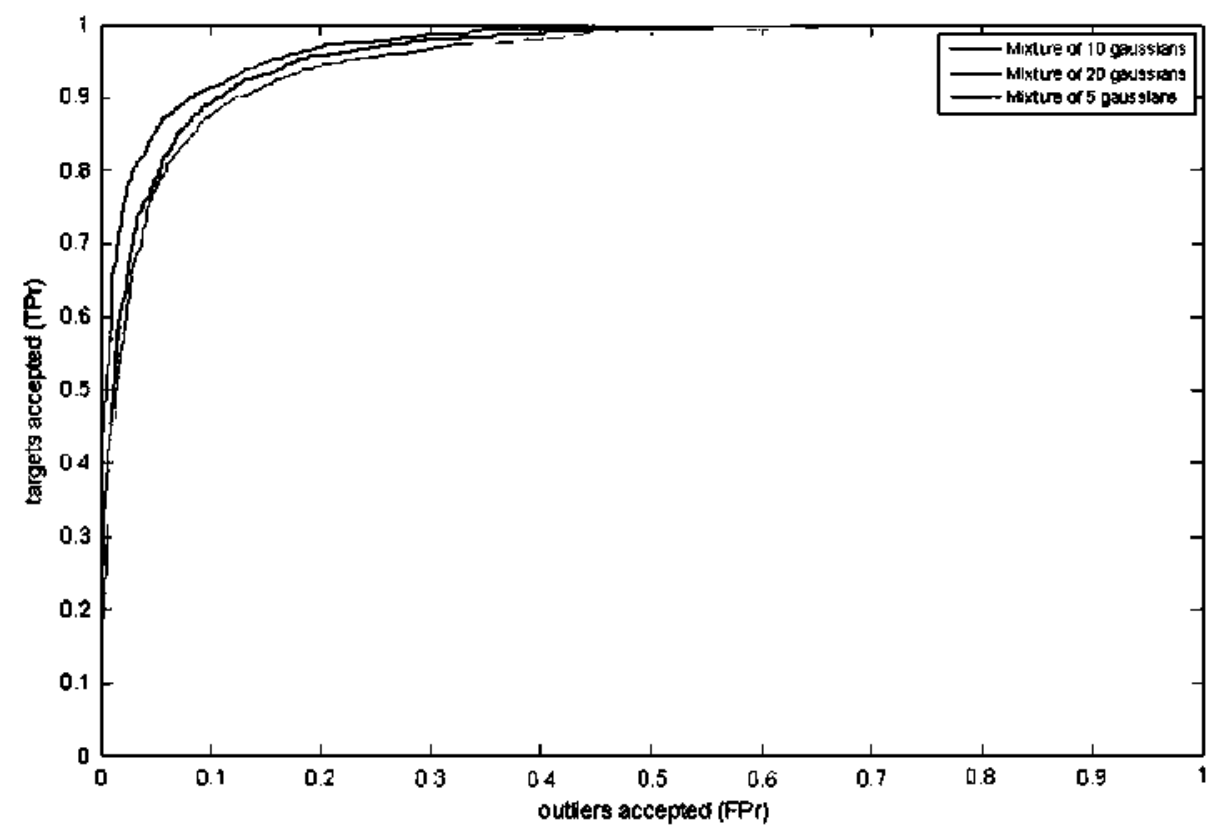

Fig. 4. ROC-curve.

- The same number of aircraft audio files was selected for every location.

- Many 'polluted' aircraft sounds were added to the outliers class dataset.

- Special attention was paid to noise sources which showed higher false positive rates (for instance, high velocity trains), including more objects for the training.

\subsection{Application}

Now, it is possible to use the ANL to detect aircraft sound events in the audio input of the system.

Other possibilities have been studied, but attending to the typical behaviour of aircraft sounds the easiest and simplest way to detect events is to apply threshold and duration criteria to the smoothed ANL signal. This way it is possible to extract small pieces of aircraft sounds in noisy environments, as the detection is only based on ANL, and it does not depend on sound pressure level time history.

Fig. 5 shows an example of the ANL time history for an aircraft sound. At the beginning and at the end of the audio file, the aircraft noise is nearly imperceptible and ANL keeps clearly below 0.5. As the aircraft gets closer to the microphone, the background noise becomes weaker compared to the foreground aircraft sound, so ANL increases to the maximum.

Fig. 6 shows the scheme we have chosen to extract aircraft noise events from the audio input, in real time.

In noisy environments, the signal-to-noise ratio (SNR, in this case, aircraft sound to background noise) may probably change over time, causing several events to be marked for the same aircraft sound event. The optimization process will extend those markers separated by $<2 \mathrm{~s}$.

During the optimization process a statistical analysis of the crisp ANL signal is made. Each marker will describe the starting and finish times, the mean ANL value, and some percentiles. The ANL90 percentile has been chosen to allow the user to include extra requirements referred to similarity.

Depending on the specific location, the background noise composition, the sound level produced by aircraft, the final user will be able to find an ANL threshold and a minimum duration that mini- mizes the number of aircraft not detected, and the number of nonaircraft detected. The user will decide how long and how polluted an event must be in order to be measured (or considered in results).

\section{Results}

All the results shown in this section have been obtained for just one training of the classifier, and using the same parameters for all measurement locations. The ANL threshold was set to 0.6 , and no statistical requirement is considered (ANL90 also 0.6). Having a previous knowledge about the acoustic environment at one specific location, would have improved the performance at that location.

Three different tests have been carried out to describe the performance of the detector:

- Events checking.

- SNR events detection.

- SNR free-run detection.

Although the system works in real time, and some specific measurements have been made after its implementation, most of the recordings were gathered at an initial stage, so it made no sense to perform real-time tests. For this reason the tests were carried out directly using the audio files.

\subsection{Events checking}

The input in this test is a single audio file which has to be classified as aircraft or non-aircraft, just depending on the detection of any aircraft-like sound longer than $d$ (seconds).

The parameter $d$ (duration) should be optimized depending on every specific location according to the SNR and the typical duration of the aircraft and non-aircraft noise events. As most environmental non-aircraft events have a short duration, $d$ can be selected as small as 5 or $10 \mathrm{~s}$, to detect aircraft that hardly exceed the background noise level. 

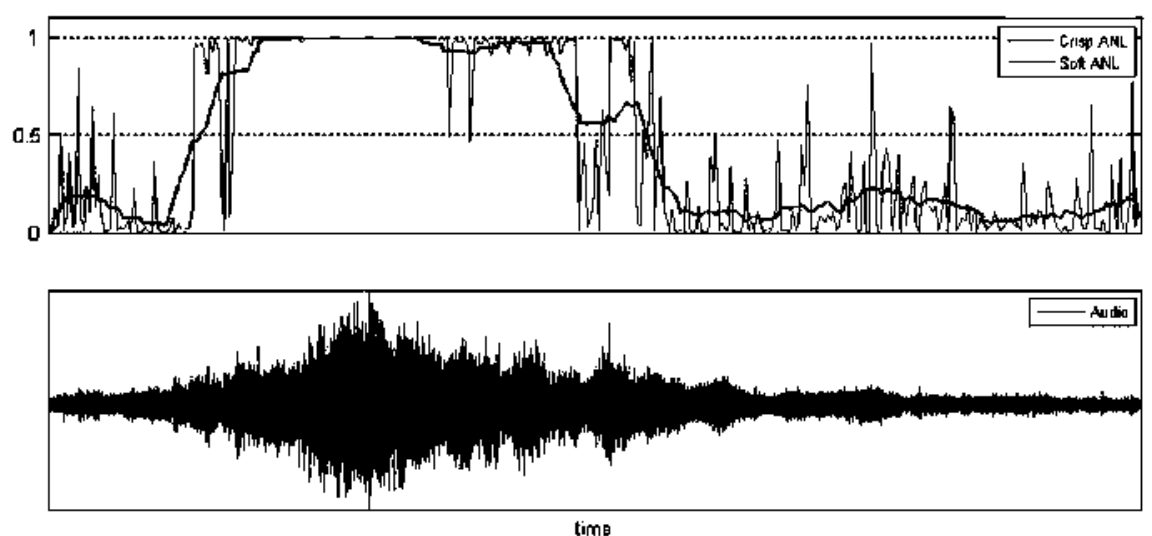

Fig. 5. Example of ANL time history for an aircraft event.

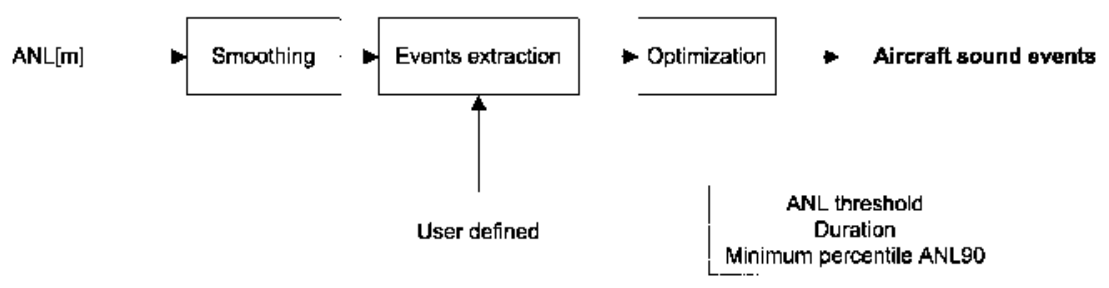

Fig. 6. Aircraft sound events extraction.

Aircraft audio files had been labeled as "pure aircraft" (negligible background noise) or "polluted aircraft" sounds (with high background noise, or superposed non-aircraft sound events).

This working mode would allow using the system with commercial noise monitors, classified previously detected events (as radar does, but based on sound characteristics).

Table 1 shows the results obtained with this test. When the user-defined searching length (d) is $5 \mathrm{~s}$, only $2.3 \%$ of the pure aircraft sounds were misclassified. Increasing $d$ to $10 \mathrm{~s}$, the false detection rates reduce to approximately $0 \%$ for most of the non-aircraft noise sources. By decreasing $d$, false detection rates will increase, and error rates of undetected aircraft events will decrease.

\subsection{SNR events detection}

All aircraft that technicians noticed during the measurements were manually marked in the measurement logs, with the help of the recordings. The one second equivalent noise level ( $L_{\text {Aeq.15 }}$ ) time history was used to estimate the background noise level for еvery single event. The noise level measured during flyover was corrected by the background noise level estimation, and then used to calculate SNR. Applying this procedure, every aircraft noise event was assigned its own SNR value.

Afterwards, the original unlabeled recordings (approximately $1 \mathrm{~h}$ length) were used for the automatic detection of aircraft, setting the duration to $5 \mathrm{~s}$. The manual and automatic lists of events were compared to obtain the results shown in Fig. 7.

\subsection{SNR free-run detection}

This test was carried out to find the relation between ANL and SNR for aircraft sounds (targets).

Only 'pure' aircraft sound events were used, as it is easy to manually decide their starting and finishing points in the recordings, and there is no doubt about the classification of all the measurements as corresponding to the target class.
Table 1

Error rates in 'events checking' test.

\begin{tabular}{lcc}
\hline & \multicolumn{2}{c}{ ANL threshold $=0.6$, ANL90 $=0.6$} \\
\cline { 2 - 3 } Noise source & $d=5 \mathrm{~s}$ & $d=10 \mathrm{~s}$ \\
\hline 'Pure' aircraft & $2.3 \%$ & $5.9 \%$ \\
'Polluted' aircraft & $23.4 \%$ & $33.9 \%$ \\
Car & $0.0 \%$ & $0.0 \%$ \\
Bus & $0.0 \%$ & $0.0 \%$ \\
Lorry & $1.0 \%$ & $0.5 \%$ \\
Motorcycle & $1.8 \%$ & $1.8 \%$ \\
Train & $2.5 \%$ & $0.6 \%$ \\
Emergency velicle & $0.0 \%$ & $0.0 \%$ \\
People & $2.9 \%$ & $0.0 \%$ \\
Animal & $1.8 \%$ & $0.0 \%$ \\
Industry & $0.0 \%$ & $0.0 \%$ \\
Machinery & $0.0 \%$ & $0.0 \%$ \\
Others & $0.0 \%$ & $0.0 \%$ \\
\hline
\end{tabular}

As only targets were under test, we then calculated ANL and SNR for every $1 \mathrm{~s}$ measurement, and then some statistics were compiled.

Background and foreground sounds cannot be simultaneously measured, so it was necessary to estimate background noise level for every single event, assuming it to be stationary throughout the whole event. As this test was only applied to 'pure' aircraft sounds, it has been considered that the background noise level during the event is equal or higher than the minimum value previous to the event. So the SNR has been slightly overestimated, which means that the results obtained are somewhat pessimistic, as the real performance of the system will be a little bit better.

Fig. 8 shows the distribution of $1 \mathrm{~s}$ measurements for the aircraft events. ANL in most of the measurements is over 0.6 , which was decided to be the threshold for classification. But when the aircraft is approaching or leaving, it sounds weaker, so SNR decreases. If $S N R$ is over $-3 \mathrm{~dB}$, the measurement will probably be classified as a target.

Fig. 9 shows the probability of a single measurement (for aircraft sounds) being misclassified as a function of SNR (ANL thresh- 


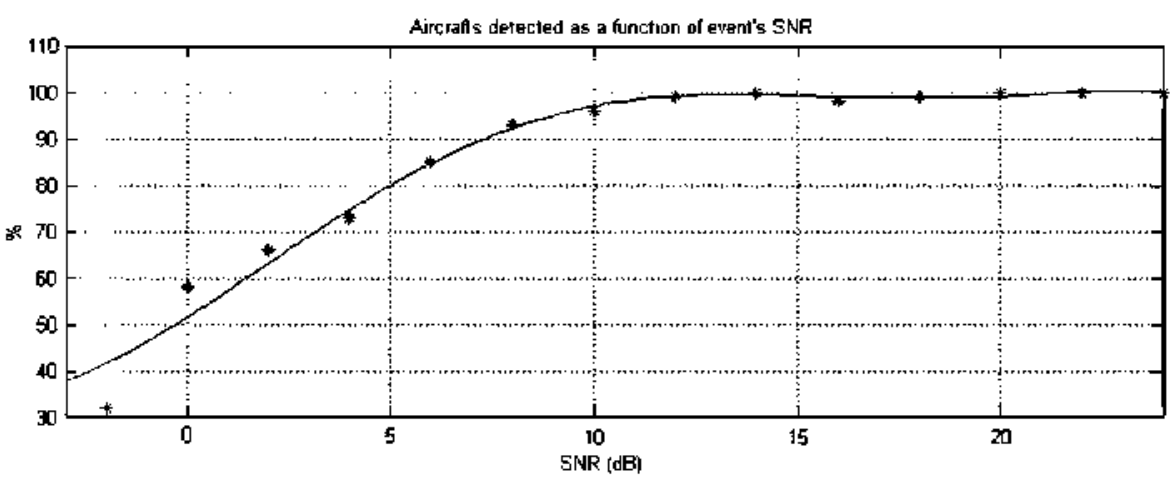

Fig. 7. Aircraft events detected as function of event's SNR.

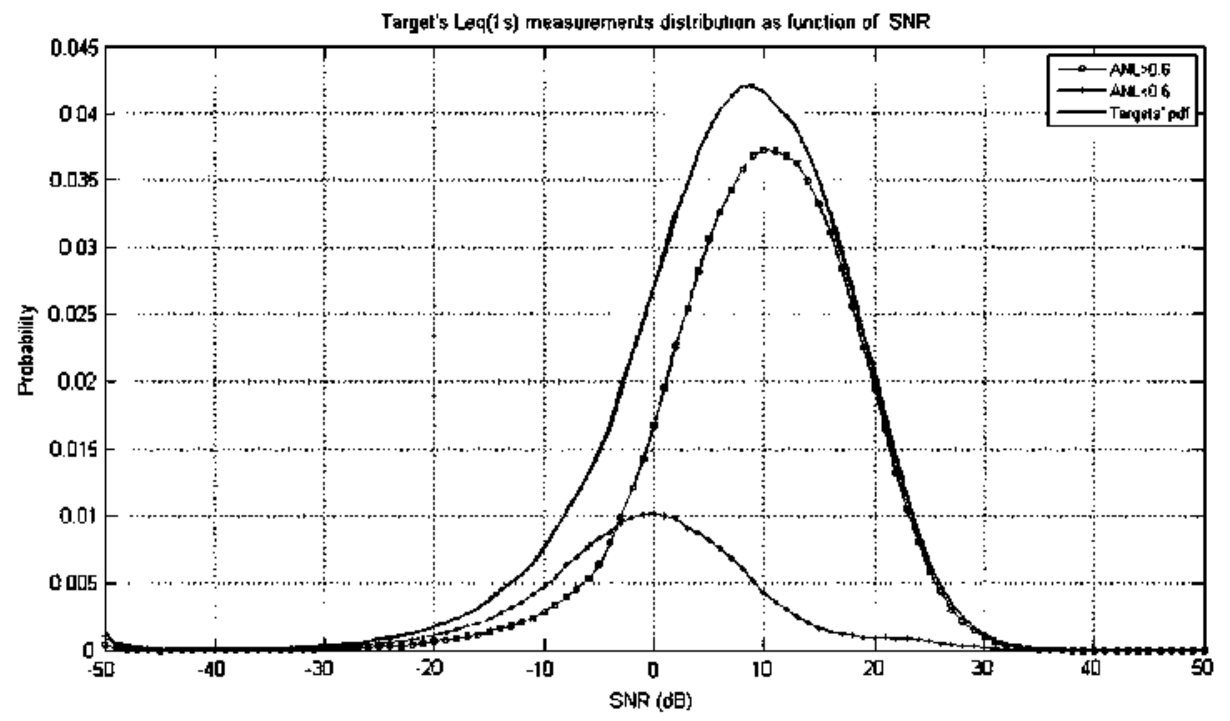

Fig. 8. Target leq [1] measurements distribution as function of 5 NR.

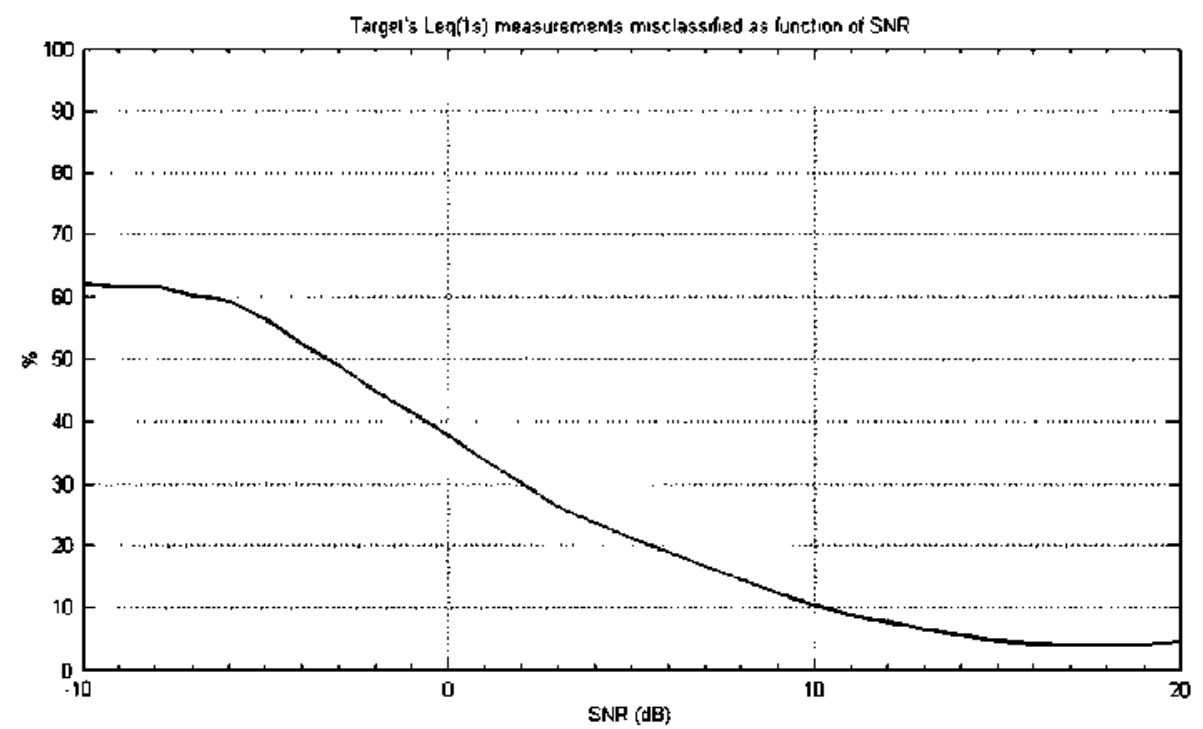

Fig. 9. Target measurements misclassified as a function of 5 NR. 
old $=0.6$ ). If SNR is over $10 \mathrm{~dB}$, the probability of error will be lower than $10 \%$, so the louder interval of the sound event will be detected.

\section{Conclusions}

An aircraft noise likeness indicator ( $A N L$ ) has been defined, and applied to the triggering of markers related to aircraft in a measurement log.

The described application of ANL to a real-time aircraft detector has shown very good results for the unattended classification of noise events in quiet environments, when SNR is big.

This application is only based on the properties of the sound, acquired with just one microphone, so it is a fairly cheap solution. Furthermore, it can be easily adapted to the detection of aircraft sound indoors, from recordings, where array-based, or radar-based systems cannot be used.

Although all the tests and training have been made using the $\mathrm{AC}$ signal from the omnidirectional microphone in the noise monitor, the use of a directional microphone could improve the results significantly (as it will improve SNR).

All the tests have been carried out for a generalized classifier and for a default setup and results have been quite good for all the selected locations. Customized setups could improve results for specific environments or locations. The system could also be easily trained for those specific environments, to show its best performance.

Future work should try to make a definition of the exact starting and finishing points of the noise events. Afterwards, the detection process could be improved in order to improve uncertainty.

The patent for the protection of the system and the algorithms described in this paper is being applied for by the Universidad Politêcnica de Madrid.

\section{Acknowledgements}

The authors are very grateful to the Ministerio de Fomento (Ministry of Public Works) for partly sponsoring this research.

\section{References}

[1] European Parliament. Directive 2002/49/EC of the European Parliament and of the Council of 25 June 2002 relating to the assessment and management of environmental noise; 2002.

[2] Design of a flight track and aircraft noise monitoring system. In: 14th International council of the aeronautical sciences, proceedings; 1984

[3] ERA - SRA international, lnc. AirScene.com NOMS.

|4| Automatic environmental noise recognition. In: IEEE international conference on industrial technology: 2004.
[5] Toyoda Y. Huang J. Ding 5X, liu Y. Environmental sound recognition by the instantaneous spectrum combined with the time pattern of power; 2004 .

[6] Cowling M, Sitte $R$ Comparison of techniques for environmental sound recognition. Pattern Recognit lett 2003:24(15):2895-907.

[7] Recognition and quantification of airclaft noise events inside dwellings. In: Inter-noise 2001 proceedings; 2001.

[8] Madras, an intelligent assistant for noise recognition. In: Proceedings internoise $97 ; 1997$.

[9] Dufournet D. Rozwadowski A. Automatic noise source recognition; 1997.

[10] Goldhor RS. Recognition of environmental sounds. Acoustics, speech, and signal processing. In: JCASSP-93, JEEE intemational conference on 1993, vol. 1;1993. p. 149-52.

|11| Automatic noise recognition in urban environments based on artificial neural networks and hidden Markov models. In: Inter-noise 2004; 2004.

|12| Couvreur C. Fontaine V. Gaunard P. Mubikangiey CG. Automatic classification of environmental noise events by hidden markov models. Appl Acoust $1998 ; 54(3): 187-206$.

[13| Aircraft sound level measurements in residential areas using sound source separation. In: Inter-noise 2004 proceedings; 2004.

|14| Cowling Michael. Non-speech environmental sound classification system for autonomous surveillance. Griffith University; 2004.

|15| Temko Andriy. Acoustic event detection and classification. Universitat Politècnica de Catalunya; 2007.

[16] Fernández L, Pogrebnyak 0 , Rodríguez ], Guerra S. Noise pattem recognition of airplanes taking off: task for a monitoring system; 2008 . p. 840 .

[17] Rojo Ruiz A, Sánchez Fernández L, Felipe-Riverón E, Suárez Guerra S. Computational model for aircraft's takeoffs pattern recognition; 2008. p. 21.

|18| Chu 5. Narayanan 5. Kuo C-J. Environmental sound recognition with timefreguency audio features audio, speech, and language processing. IEEE Trans $20092009 ; 17(6): 1142-58$.

[19] Genescà M, Romeu J, Pàmies T, Sánchez A. Real time aircraft fly-over noise discrimination. J Sound Vib 2009;323(1-2):112-29.

[20] Zadel LA. Fuzzy sets. Inf Control 1965:8(3):338-53.

[21] Hodgdon KK, Atchley AA, Bernhard R]. Low frequency noise study. In: PARTNER-COE-2007-001; 2007

[22] Slaney M. Auditory toolbox, version 2; 1998.

[23] Duin RPW. Pattem recognition tools - Prtools 4.1.4; 2009

24| Duda R. Hart P. Pattern classification and scene analysis; 1973.

[25] Intemational Neural Network Society (INNS), editor. One-class classifier networks for target recognition applications. Proceedings world congress on neural networks; 1993.

[26] Ritter G, Gallegos Ma. Outliers in statistical pattern recognition and an application to automatic chromosome classification. Pattern Recognit Lett $1997 ; 18(6): 539$.

|27| David MJ Tax. One-class classification; concept-learning in the absence of counter-examples. ln: ASCl dissertation series, vol. 65. Delft University of Technology; 2001.

|28| Rabaoui A, Davy M, Rossignol 5, lachiri Z, Ellouze N. Improved one-class 5VM classifier for sounds classification. In: IEEE Conference on 2007. Advanced video and signal based surveillance, AVSS 2007; 2007, p. 117-22.

|29| Face detection using one-class-based support vectors. In: Sixth IEEE intemational conference on automatic face and gesture recognition; 2004

[30] Tax DM]. Data description toolbox - dd_tools 1.6.3; 2008

[31] Cohn David, Atlas Les, Ladner Richard. Improving generalization with active learning. Mach Learn 1994;15:201-21.

|32| Cebron N. Berthold $M$. Active learning for object classification: from exploration to exploitation. Data Min Knowl Disc 2009;18(2):299.

|33| Juszczak P. Duin R. Selective sampling methods in one-class classification problems: artificial neural networks and neural information processing. In: ICANN/ICONIP 2003, 03-09; 2007. p. 140-8. 